\title{
Fasciotomy in compartment syndrome from snakebite
}

\author{
Yong Hun Kim, Jin-hee Choi, Jiye Kim, Yoon Kyu Chung \\ Department of Plastic and Reconstructive Surgery, Wonju Severance Christian Hospital, Yonsei University Wonju College of Medicine, Wonju, \\ Korea
}

Background Local symptoms and signs of snake envenomation mimic the clinical features of compartment syndrome. It is important to measure the intracompartmental pressure to diagnose compartment syndrome. In this study, we present our experiences of confirming compartment syndrome and performing fasciotomy in snakebite patients based on high intracompartmental pressure findings.

Methods The medical records of patients who visited the trauma center of Wonju Severance Christian Hospital from January 2010 to December 2015 for the management of venomous snakebite were retrospectively reviewed. Starting in 2014 , fasciotomy was performed in patients with an intracompartmental pressure of more than $40 \mathrm{mmHg}$ in addition to the clinical symptoms of compartment syndrome.

Results A total of 158 patients with snakebite came to the hospital within 48 hours for treatment. Most patients (110 patients) were bitten at the upper extremities (69.6\%). Since 2014, 33 out of 59 patients were suspected to have compartment syndrome, and their intracompartmental pressures were measured. Seventeen of those patients had a high intracompartmental pressure (average, $49.6 \mathrm{mmHg}$; range, $37-88 \mathrm{mmHg}$ ), and fasciotomy was performed. Conclusions In this study, as many as $10.8 \%$ of all cases were in need of fasciotomy when compartment syndrome was diagnosed by measuring the intracompartmental pressure. Previously, it was reported that fasciotomy was not required in many cases of compartment syndrome originating from snakebite. However, some patients may develop very severe compartment syndrome, requiring fasciotomy.

Keywords Snake bites / Compartment syndromes / Fasciotomy
Correspondence: Yoon Kyu Chung Department of Plastic and Reconstructive Surgery, Wonju Severance Christian Hospital, Yonsei University Wonju College of Medicine, 20 Ilsan-ro, Wonju 26426, Korea Tel: +82-33-741-0611

Fax: +82-33-732-4022 E-mail: ykchung@yonsei.ac.kr

Received: 25 May $2018 \bullet$ Revised: 24 Oct $2018 \bullet$ Accepted: 24 Oct 2018

pISSN: 2234-6163 • elSSN: 2234-6171 • https://doi.org/10.5999/aps.2018.00577• Arch Plast Surg 2019;46:69-74

\section{INTRODUCTION}

Snakebite is a specialized form of injury that occurs throughout the world, most frequently in the countryside. Depending on the snake species, the injected venom leads to a wide variety of toxic effects, ranging from local tissue damage to systemic organ failure. Although antivenom is effective in preventing or limiting the systemic effects, the management of local symptoms remains problematic, especially in cases of soft tissue necrosis and compartment syndrome. The local symptoms and signs of snake envenomation include severe pain and the appearance of an immobile, tensely-swollen, cold, and apparently pulseless extremity. Clinically, this collection of cytotoxic symptoms can be indistinguishable from concomitantly developing compartment 
syndrome. In the literature, however, the management of compartment syndrome in such cases is not clear. The main objective of this study was to review patients' outcomes following snakebite and to highlight the need for measuring the intracompartmental pressure.

\section{METHODS}

A single-institutional retrospective review was performed for all patients who presented after being bitten by venomous snakes between January 2010 and December 2015. This study was approved by the Institutional Review Board (CR318101). A patient was considered to have suffered a snakebite only if the site of injury contained a pair of puncture marks consistent with fang marks. Patient charts were reviewed for demographics, the physical location where the patients were bitten, the anatomic site of the bite, the season, the time from snakebite to presentation, the need for an antivenin, surgical intervention, and complications. Patients were excluded if they had been treated at another institution prior to being transferred to Wonju Severance Christian Hospital and if they presented more than 48 hours after the initial snakebite.

Patients presenting to our institution were managed according to the World Health Organization (WHO) guideline for the clinical management of snakebite in Southeast Asia [1]. The primary assessment included measurements of vital signs and an examination of the bite wound. A laboratory examination was conducted, consisting of a complete blood count, general chemistry, coagulation profile, and creatinine kinase. Antivenom was administered for severe local tissue damage or clinical signs and laboratory evidence of systemic derangement.

The antivenom was prepared by mixing a vial of antivenom powder effective against Gloydius blomhoffii brevicaudus (KoVax Dried Antivenom Injection, Korea Vaccine Co. Ltd., Ansan, Korea). A vial contains 6,000 units, which is dissolved in a total volume of $10 \mathrm{~mL}$ of normal saline. The skin reaction was observed by injecting $0.1 \mathrm{~mL}$ of the antivenom solution into the subcutaneous tissue. Antivenom administration was abandoned if erythema or swelling greater than $1 \mathrm{~cm}$ or symptoms suggestive of an anaphylactic response were observed. In the presence of an equivocal response, an additional $0.9 \mathrm{~mL}$ of the solution was administered subcutaneously, with the patient kept under observation for 30 more minutes. The initial antivenom dose was 6,000 $\mathrm{IU}$ in most cases. Patients experiencing a severe cytotoxic response were administered 12,000 IU of antivenom.

Between 2010 and 2013, compartment syndrome was diagnosed based on a clinical assessment. In 2014 and 2015, all patients who were clinically suspected to have compartment syndrome were diagnosed using intracompartmental pressure measured via a cannula introduced into the compartment (Fig. 1). The intracompartmental pressure was measured every four hours while the patient was in the emergency department and continued in the ward until symptoms resolved or the pressure readings warranted an operation. Fasciotomy was performed for patients with an intracompartmental pressure greater than 40 $\mathrm{mmHg}$ [2] who did not demonstrate a pressure decrease at 4 hours after antivenom administration.

\section{RESULTS}

During the 6-year period reviewed, 168 patients presented for snakebite. Of these, 10 patients were excluded either because they were transferred from another hospital or because they presented more than 48 hours after the initial snakebite $(n=$

\section{Fig. 1. Intracompartmental pressure measurement}

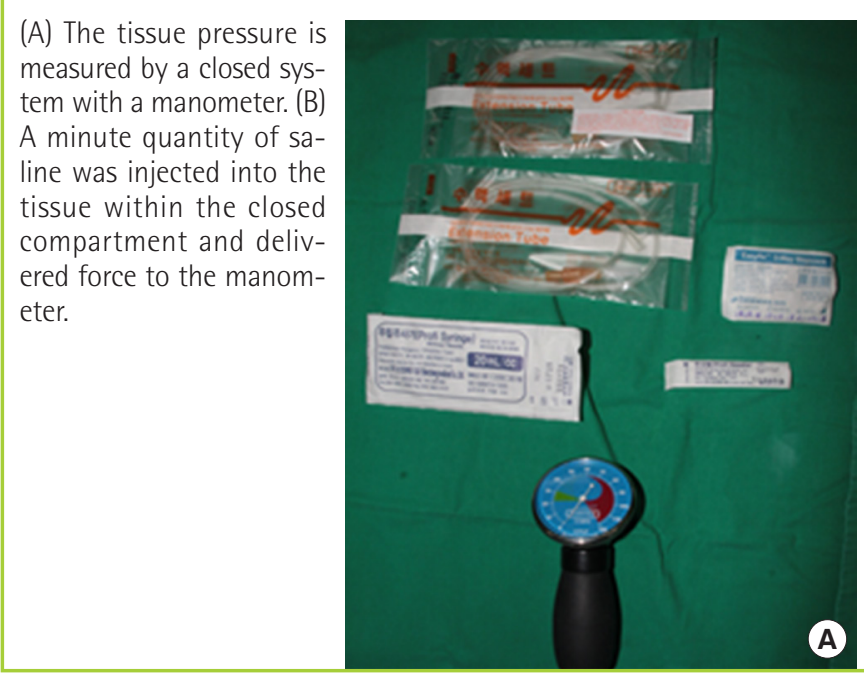

(A) The tissue pressure is measured by a closed system with a manometer. $(B)$ A minute quantity of saline was injected into the tissue within the closed compartment and delivered force to the manom eter. 
Table 1. Age and sex distribution of patients

\begin{tabular}{|lccc|}
\hline \multirow{2}{*}{ Age $(\mathrm{yr})$} & \multicolumn{2}{c}{ No. of patients } & \multirow{2}{*}{ Total $(\%)$} \\
\cline { 2 - 3 } & Male & Female & \\
\hline $1-20$ & 8 & 2 & $10(6.3)$ \\
$21-30$ & 5 & 3 & $8(5.1)$ \\
$31-40$ & 7 & 1 & $8(5.1)$ \\
$41-50$ & 13 & 9 & $22(13.1)$ \\
$51-60$ & 30 & 15 & $45(28.5)$ \\
$61-70$ & 20 & 16 & $36(22.8)$ \\
$>70$ & 19 & 10 & $29(18.4)$ \\
Total & 102 & 56 & $158(100.0)$ \\
\hline
\end{tabular}

158). Men represented most of the patients (102 males vs. 56 females) (Table 1). The upper extremity was the most common site of snakebite $(110 / 158,70 \%)$ (Table 2). Snakebites were more common during the summer months, with $55 \%$ occurring in July and August. The most common time of the day for snakebite was between midday and $6 \mathrm{PM}$, which corresponds to the peak outdoor activity time. The snakebite-to-hospital time ranged from less than 30 minutes to 24 hours. Only three out of 158 patients presented to the hospital between 24 and 48 hours after the snakebite. Excluding these three outliers, the mean snakebite-to-hospital time was 6 hours for the remainder of patients. The mean length of hospital stay was 7.4 days. Antivenom was administered to 102 patients (64.6\%), with the mean dose being 1.48 vials per patient (6,000 IU/vial). Of the 158 patients, 59 patients were managed at our institution starting in 2014, when we began measuring the intracompartmental pressure in patients suspected of having compartment syndrome. Among these 59 patients, 33 had a clinical presentation suspicious of compartment syndrome and underwent an intracompartmental pressure measurement. Ultimately, intracompartmental pressure monitoring identified 17 patients who met the objective criteria for compartment syndrome, in whom fasciotomy was performed. In those 17 patients, the mean intracompartmental pressure was $49.6 \mathrm{mmHg}$ (range, 37-88 $\mathrm{mmHg}$ ). In the other patients, either the measured pressure was not very high, or the pressure was initially high, but it markedly decreased during the observation period.

\section{DISCUSSION}

In the past decade, snakebites have been verified as a common occupational hazard for farmers and field workers and have been officially recognized by the $\mathrm{WHO}$ as a neglected tropical disease $[3,4]$. In addition to being an occupational hazard for workers in developing countries, snakebites are becoming more common in developed countries with improvements in quality
Table 2. Affected anatomic sites and cases in which fasciotomy was performed

\begin{tabular}{|lcc|}
\hline Anatomic site & No. (\%) & Fasciotomy \\
\hline Finger & $64(41)$ & 7 \\
Hand & $33(21)$ & 4 \\
Foot & $21(13)$ & 3 \\
Toe & $15(10)$ & 1 \\
Forearm & $13(8)$ & 2 \\
Lower leg & $7(4)$ & 0 \\
Thigh & $5(3)$ & 0 \\
Total & $158(100)$ & 17 \\
\hline
\end{tabular}

of life, as spending more leisure time in outdoor activity equates to an increased chance of snake encounters and, in turn, snakebites. Worldwide, the estimate of snakebite victims is 421,000 persons per year, of whom 20,000 people die as a complication of the envenomation event [5]. In South Korea, the annual incidence of snakebites ranges from 192 to 621 cases, with an average of five deaths per year. There are four species of venomous snakes that reside in Korea: Gloydius blomhoffii, Gloydius saxatilis, Gloydius ussuriensis, and Rhabdophis tigrinus [6]. The venom from these snakes is usually not toxic, and systemic derangement from the response to the injected toxin is rare. Only a few clinical cases of mortality have been documented on the Korean peninsula. Consistent with other reports, our study found the same epidemiologic patterns, in which most bites happened in the afternoon and evening and the peak incidence of bites occurred in the rainy season $[7,8]$. In contrast to reports from Southeast Asian countries, where $70 \%$ of injuries are to the lower extremity, the percentage of upper extremity injuries is $69.6 \%$ in Korea. Although systemic complications, such as rhabdomyolysis, acute renal failure, coagulopathy, and hemolytic anemia were observed, they did not lead to mortality [9]. In most cases, the symptoms as well as the lab processes were improved by the use of antivenom and conservative treatment. However, in addition to systemic complications, local complications, such as soft tissue necrosis and compartment syndrome, remain problematic. Hematotoxic/cytotoxic venom can initiate a significant amount of inflammation and edema in the injected tissue. If this process occurs deep within a compartment surrounded by noncompliant tissue (i.e., bone, ligament, and intermuscular fascia), the increasing edema leads to rising compartmental pressure and the potential for obstruction of distal perfusion, as well as local tissue ischemia. Although compartment syndrome has the same underlying physiologic basis regardless of the initial cause, the clinical presentation and diagnosis differ significantly between cases of snake envenomation and high-energy injury. In high-energy trauma (i.e., crush injuries), the clinical presenta- 
tion alone is often sufficient to distinguish the physiologic changes from the injury itself from these of compartment syndrome (e.g., pulselessness and pain out of proportion to the injury). However, these distinguishing features of compartment syndrome are not reliable indicators in cases of snakebite because snake venom itself can cause hemodynamic instability (pulselessness) and significant pain that is out of proportion to two mere fang marks. The potential for the missed diagnosis of compartment syndrome in cases of snakebite was initially responsible for liberal use of fasciotomy in the 1960s for the management of snakebites, when a low threshold was set for snakebite-related compartment syndrome. The ischemic signs of compartment syndrome, excluding severe pain, are late signs and should not be relied on for the early diagnosis of compartment syndrome [10]. For the past decades, the clinical consensus has been that such a low threshold was a cause of unnecessary surgical interventions, and various authors have advocated the use of antivenom in snakebite injuries, with fasciotomy performed under strict, absolute indications [11]. Currently, the established guidelines suggest that fasciotomy is indicated in snake-bitten limbs that meet the following criteria $[1,12,13]$ :
(1) hemostatic abnormalities have been corrected (antivenom with or without clotting factors); (2) clinical evidence of intracompartmental syndrome; or (3) intracompartmental pressure $>40 \mathrm{mmHg}$ (in adults).

The incorporation of intracompartmental pressure measurements as a diagnostic criteria for snakebite-related compartment syndrome is relatively new. So far, the authors are not aware of any other study on the use of objective pressure data to evaluate the need for fasciotomy in the context of snakebites. Between 2010 and 2013, fasciotomies were performed in just two cases at our institution, consistent with other studies reporting single cases of fasciotomy among hundreds of snakebite patients [1416]. In 2014, intracompartmental pressure measurements were adopted as an official policy at our institution for patients suspected of having compartment syndrome from snakebites $(\mathrm{n}=59)$. This change led to pressure readings in 33 patients, of whom 17 ultimately required fasciotomy $(17 / 158,10.8 \%)$ for persistently elevated pressure readings despite antivenom administration. In comparison to the reported rates, the frequency of fasciotomy at our institution appears to be extremely high. One explanation for this may be the mean time interval of 9.8

Fig. 2. Case of fasciotomy

(A) A 65-year-old woman was bitten from snake on the right second finger. (B) After few hours later, painful swelling with paresthesia was observed on the fingers and hand. (C) Fasciotomy was performed on dorsal side of the hand, immediately postoperative image. (D) One day after the operation, symptoms were significantly decreased. (E) Two weeks after postoperatively, symptoms almost relief and wound healing by secondary intention.
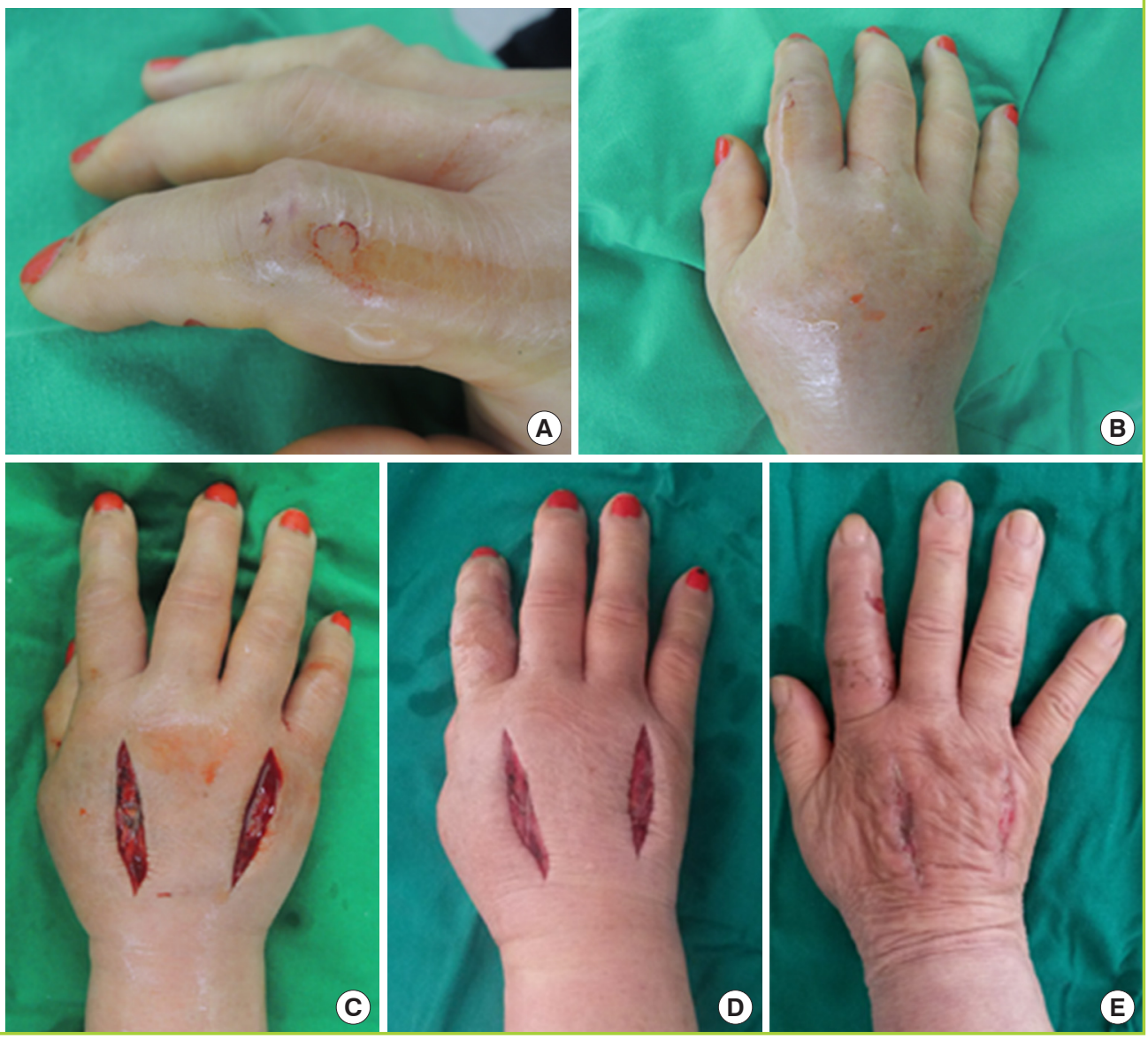
hours (range, 1-28 hours) between snakebite and antivenom administration, which is significantly longer than the recommended 3-hour window. Another potential reason for the high rate of fasciotomy at our institution may be related to the higher rate of upper extremity involvement. In other reports, the lower extremity was more frequently involved than upper extremity (i.e., the hands). This can be a significant distinction, as the foot is usually protected by footwear, and snake fangs are generally not long enough to deliver the venom directly into the deeper compartments of the lower leg. Unless venom is injected deeper, directly into the muscular compartment, most swelling therefore occurs outside of the compartment and rarely impairs intracompartmental perfusion pressure. In the hand and forearm, compartment syndrome is more likely because snakebites can often lead to venom delivery into deep compartments [17]. In our study, 12 out of 17 fasciotomies were performed for snakebites on the hand, which suggests the anatomic site may be an important factor contributing to the development of compartment syndrome in snakebite (Fig. 2). An alternative interpretation of our study is that, prior to 2013, we may have been too conservative in the management of potential compartment syndrome in snakebite wounds. Because both compartment syndrome and hematotoxic envenomation cause tissue necrosis, we may have missed the opportunity to perform fasciotomy in patients with actual compartment syndrome. In these patients, the tissue necrosis from increased tissue pressure and ischemia could easily have been considered as the consequence of hematotoxic venom.

\section{NOTES}

\section{Conflict of interest}

No potential conflict of interest relevant to this article was reported.

\section{Ethical approval}

The study was approved by the Institutional Review Board of Wonju Severance Christian Hospital (IRB No. CR318101) and performed in accordance with the principles of the Declaration of Helsinki. Written informed consents were obtained.

\section{Patient consent}

The patients provided written informed consent for the publication and the use of their images.

\section{ORCID}

Yong Hun Kim https://orcid.org/0000-0002-6092-4374

Jin-hee Choi https://orcid.org/0000-0003-0414-0679
Jiye Kim https://orcid.org/0000-0002-1724-4250

Yoon Kyu Chung https://orcid.org/0000-0002-0401-3912

\section{REFERENCES}

1. Warrell DA. Guidelines for the management of snake-bites [Internet]. New Delhi, World Health Organization: c2010 [cited 2018 Dec 5]. Available from http://apps.searo.who. int/PDS_DOCS/B4508.pdf.

2. Whitesides TE, Haney TC, Morimoto K, et al. Tissue pressure measurements as a determinant for the need of fasciotomy. Clin Orthop Relat Res 1975;113:43-51.

3. World Health Organization. Rabies and envenomings: a neglected public health issue. Report of a consultative meeting [Internet]. Geneva, WHO: c2007 [cited 2010 Feb 8]. Available from http://www.who.int/bloodproducts/animal_ sera/Rabies.pdf.

4. Williams D, Gutierrez JM, Harrison R, et al. The Global Snake Bite Initiative: an antidote for snake bite. Lancet 2010; 375:89-91.

5. Kasturiratne A, Wickremasinghe AR, de Silva N, et al. The global burden of snakebite: a literature analysis and modelling based on regional estimates of envenoming and deaths. PLoS Med 2008;5:e218.

6. Lim H, Kang HG, Kim KH. Antivenom for snake bite in Korea. J Korean Med Ass 2013;56:1091-103.

7. Laohawiriyakamol S, Sangkhathat S, Chiengkriwate P, et al. Surgery in management of snake envenomation in children. World J Pediatr 2011;7:361-4.

8. Jang IS, Lee JA, Kim SY, et al. Clinical features in snake bite. J Korean Soc Emerg Med 1996;7:580-9.

9. Jin SC, Lee JW, Yang SJ, et al. Consideration of factors associated with complications and systemic symptoms of snake bites. J Korean Soc Emerg Med 2008; 19:686-96.

10. Hsu CP, Chuang JF, Hsu YP, et al. Predictors of the development of post-snakebite compartment syndrome. Scand J Trauma Resusc Emerg Med 2015;23:97.

11. Chattopadhyay A, Patra RD, Shenoy V, et al. Surgical implications of snakebites. Indian J Pediatr 2004;71:397-9.

12. Hall EL. Role of surgical intervention in the management of crotaline snake envenomation. Ann Emerg Med 2001;37: 175-80.

13. Gold BS, Dart RC, Barish RA. Bites of venomous snakes. N Engl J Med 2002;347:347-56.

14. Watt CH Jr. Treatment of poisonous snakebite with emphasis on digit dermotomy. South Med J 1985;78:694-9.

15. Wingert WA, Chan L. Rattlesnake bites in southern California and rationale for recommended treatment. West J Med 
1988; 148:37-44.

16. Russell FE, Carlson RW, Wainschel J, et al. Snake venom poisoning in the United States. Experiences with 550 cases. JAMA 1975;233:341-4.
17. Nuchprayoon I, Pongpan C, Sripaiboonkij N. The role of prednisolone in reducing limb oedema in children bitten by green pit vipers: a randomized, controlled trial. Ann Trop Med Parasitol 2008;102:643-9. 